Electronic version of an article published as [International Journal of Production Economics, 2006, Vol. 103, No. 1, p. 3-9]

[DOI: http://dx.doi.org/10.1016/j.ijpe.2005.05.015] @ [copyright Elsevier] 


\title{
Rotational allocation of tasks to multifunctional workers in
}

\author{
a service industry ${ }^{*}$
}

\author{
Albert Corominas ${ }^{\mathrm{a}}$, Rafael Pastor ${ }^{\mathrm{a}}$ and Ericka Rodríguez ${ }^{\mathrm{a}}$ \\ ${ }^{a}$ IOC Research Institute, Universitat Politècnica de Catalunya, Avda. Diagonal 647, pl. 11, 08028 Barcelona, Spain
}

\begin{abstract}
This paper deals with the assignment of tasks to the members of the multi-functional staff (each worker is able to perform a given subset of types of tasks) of a work centre, during each period (e.g. 1 h) into which the planning horizon (e.g. 1 shift or 1 week) can be divided. For each type of task to perform, all workers who can perform the task do so at equal worker efficiencies. There are constraints that, if possible, should be respected. The objective is that the percentage of working time dedicated by each worker to each type of task be as close as possible to reference values. The problem is modelled as a sequence of assignments, in which appropriate values for the cost matrix depend on the results of the previous assignments. The obtained results are satisfactory: the solutions meet the constraints, the scheduled percentages steadily approach reference values and the calculation times are very short. Therefore, the work presented constitutes a potential tool for assigning tasks to multi-functional workers in the service industry.
\end{abstract}

Keywords: Manpower planning; Job rotation; Heuristics; Assignment problem

${ }^{*}$ This research has been supported by the Spanish MCyT projects DPI2001-2176 and DPI2004-05797, co-financed by FEDER. 


\section{Introduction}

At the present, the organisation of working time (e.g. Corominas and Crespán, 1993) is a fundamental tool for increasing productivity (e.g. Cox, 1989). Working time flexibility (Oke, 2000) and the multi-functionality of workers helps to mould production capacity more closely to demand.

Several authors (e.g. Abernathy et al., 1973 and Siferd and Benton, 1992) present a hierarchical scheme for work force organisation problems that consists of three phases: (1) planning (Corominas et al., 2004); (2) scheduling (e.g., Lagodimos and Leopoulos, 2000, Bellanti et al., 2004, Cappanera and Gallo, 2004 and Demerouti et al., 2004); (3) allocation. The assignment of tasks to multi-functional workers is done during phase (3), once a schedule has been assigned to each worker.

Even though in the past the assumption held that workers could only perform one type of task (Buffa et al., 1976), at the present worker multi-functionality is often assumed. Due to the multifunctionality, the possibilities to organize the workforce increase, and this has an important effect upon labour cost (Zülch et al., 2004). Bergman (1994) highlights the importance of multifunctionality of hospital staff (each worker being able to perform a given subset of types of tasks), based on individual experience. In Corominas et al. (2002), a problem of planning staff working hours over an annual horizon is solved by assuming multi-functionality and an equal efficiency for all the members of the staff capable to perform a type of task. Campbell and Diaby (2002) presents a multi-department, labour-intensive service environment for allocating tasks to cross-trained workers, such as that faced by hospital nurses; although the authors assume that each worker will perform the same task during the whole shift, they suggest the possibility of considering the reassignment of tasks to workers within the shifts. Tharmmaphornphilas and Norman (2004) discuss the advantages of rotating tasks to reduce worker fatigue and injuries.

In this paper, we deal with the problem of allocating types of tasks to the multi-functional workers of a service centre over a time horizon. A type of task must be assigned to each worker for each of the periods (e.g., $1 \mathrm{~h}$ ) into which the planning horizon (e.g. 1 shift or 1 week) can be divided. We assume that worker efficiencies are equal for all those that can perform a given type of task. In addition, for a given period it is assumed that the set of workers present and the working capacity necessary for each type of task is known. The objective is that the percentage of time dedicated by workers to each type of task be as close as possible to established ideal values, which may or may not be equal for all of them. There exist additional constraints that should be respected if possible, such as the maximum or minimum number of periods during which one can continuously perform the same type of task, or the minimum number of periods to go back and start with the same type of task.

The problem presented in this paper generalises a task allocation problem that was presented to us by a service company. The study company is a retail chain that sells clothes. This company began its activities in the 1950s; nowadays it has around 800 boutiques in 15 countries and more than 30,000 employees; its annual turnover goes beyond the 1,500 million of euros. In that case:

- Each shop's staff (excluding the heads of the sections) was completely multi-functional (i.e. capable of performing any type of task). 
- The management's criteria stipulated that the proportion of time dedicated by workers to each type of task should be the same for all workers (to keep workers trained for every task and also for reasons of equity, since different tasks required varying levels of attention and responsibility).

- Provided that it was possible, the following constraints were also to be fulfilled: (i) the number of consecutive periods dedicated by a worker to a given type of task should fall within to a specified interval limited by a minimum and a maximum number of periods working at each type of task; and (ii) once workers had worked at a specific type of task for a certain number of consecutive periods, a minimum number of periods would have to pass before they could perform the same type of task again.

This particular case illustrates a more general situation that can appear in a great variety of companies, especially in the service industry.

The rest of the article is organised as follows: the allocation problem is modelled and solved as a sequence of assignment problems in Section 2; Section 3 includes one example; Section 4 contains the results of a computational experiment and Section 5 the corresponding conclusions.

\section{Model and resolution}

The procedure that is proposed for assigning the tasks to be performed to available workers consists in solving a sequence of assignment problems: one is solved for each period $t$ in the planning horizon, $T$, in chronological order. The elements of the assignment matrices are calculated so that the solution obtained presents the following desired characteristics: the percentages of working time dedicated to different types of tasks are near the ideal values; and, if it is possible, the conditions specified are met.

Worker efficiencies are assumed to be equal for all workers who can perform a given type of task.

In Corominas et al. (2005), a procedure is proposed for calculating the number of workers in each category $c$ that have to perform each type of task $k$ for every period $t$ in planning horizon $T$. The objective function includes penalties assigned to shortages and surpluses of capacity.

Thus, it can be assumed that during each period, as the result of the previous phases of the hierarchical procedure, the number of available workers is equal to the number of "units of work" (considering a unit of work to be the performance of a specific type of task during one period) and, in the case of partial multi-functionality, that a feasible assignment exists.

For each period $t$ in planning horizon $T$, the set of available workers and the number of units of work needed for each type of task are known.

The proportion of time dedicated by each worker $i$, during a determined time interval (e.g. 1 year or 1 semester), to a task $k$, that he is trained to perform, should be as close as possible to the ideal reference value $\left(\mathrm{PT}_{i k}\right)$.

Some assignments have to fulfil specific conditions. For instance, the following conditions were required by a given service company: (i) lower and upper bounds on the number of consecutive periods dedicated by workers to the same type of task; (ii) a minimum number of periods that must pass before a worker can perform the same type of task again. Of course, other conditions may be 
in force in other companies. In every case, we assume that the conditions are soft constraints, in the sense that they must be satisfied if at all possible; however, in order to obtain a practicable solution, they may be violated.

For each period $t$ (e.g. $1 \mathrm{~h}$ ) of the planning horizon $T$, the data are the following:

$W \quad$ set of multifunctional workers available to be assigned $(i=1, \ldots,|W|)$.

$C \quad$ number of categories of workers $(c=1, \ldots, C)$.

$\mathrm{Cat}_{i} \quad$ category of worker $i(i=1, \ldots,|W|)$.

$K \quad$ number of types of tasks $(k=1, \ldots, K)$.

$F$

matrix whose elements, $f_{c k}$, are equal to 1 iff the workers of category $c(c=1, \ldots, C)$ can perform tasks of type $k(k=1, \ldots, K)$

integer number of workers (capacity) that should be assigned to task

$D_{k}$

type $k(k=1, \ldots, K)$. These values must fulfil the condition $\sum_{k=1}^{K} D_{k}=|W|$.

ideal proportion of working time that a worker $i$ should dedicate to performing a task of type $k\left(\forall i, \forall k \mid f_{\text {Cat }_{i}, k}=1\right)$.

$\mathrm{PT}_{i k}$

Of course, when defining values for these parameters, one must take into account the forecasted demand for each type of task.

relative importance of adjusting worker $i$ and the type of

$\alpha_{i k}$ $\operatorname{task} k\left(\forall i, \forall k \mid f_{C a t_{i}, k}=1\right)$ to the value $\mathrm{PT}_{i k}$.

$H_{i}$ is the number of past periods that are taken into account for computing the actual proportion of worker $i$ 's time dedicated to each type of task. $H_{i}$, Hist $_{i k}$ Hist $_{i k}$ is the number of periods, within $H_{i}$ previous periods, in which worker $i$ has performed tasks of type $k$. 
$\mathrm{NPS}_{i k}$

number of consecutive periods immediately prior to the current

period during which worker $i$ has performed tasks of type $k$.

number of consecutive periods immediately prior to the current

$\mathrm{NPP}_{i k}$

period during which worker $i$ has not performed tasks of type $k$.

number of continuous periods during which worker $i$ is present, starting at $t$ (included), and continuing until the first period of the

$\mathrm{NPF}_{i}$ worker's absence (because of a break, lunch or off shift) or until the end of planning horizon $T$.

minimum number of consecutive periods that one worker should work

$\operatorname{NMin}_{k}$ at a task of type $k$.

maximum number of consecutive periods that one worker can work at $\operatorname{NMax}_{k}$ a task of type $k\left(\mathrm{NMax}_{k} \geqslant \mathrm{NMin}_{k}\right)$.

minimum number of consecutive periods that a worker should refrain $\mathrm{NPer}_{k}$ from performing a task of type $k$ before performing it again.

The problem could be modelled using a mathematical program, but this would result in a very high number of integer variables and constraints and a non-linear objective function. Therefore, another approach is proposed: that of solving a sequence of assignment problems (one for each period $t$ in the planning horizon $T$, in chronological order). The values of the elements of the assignment matrix that correspond to period $t$ are then calculated as a function of the results of these assignments.

The assignment matrix corresponding to a specific period has a row for each worker $i$ at the company and $D_{k}$ columns for each type of task $k$. The elements $C_{i j}$ of the matrix take into account the consequences of assigning worker $i$ the type of task corresponding to column $j$.

From the outset, the elements of the matrix have to reflect, as a benefit or as a cost, respectively, the fact that the assignment implies an approximation to or a deviation from the ideal values for the proportion of working time that each worker dedicates to each type of task. Moreover, the solution must fulfil, if it is possible at all, the conditions sought by the company (thus, the assignments that are not compatible with these conditions are penalised with a very high value, $M$ ). Of course, the 
assignment of a worker to a type of task that he or she is not able to perform $\left(f_{\text {Cat }_{i}, k}=0\right)$, as this must be considered strictly forbidden, is penalised with a higher order value $\bar{M}(\gg>M)$.

The elements $C_{i k}$ of the assignment matrix $\left(\forall k \mid f_{C a t_{i}, k}=1\right)$ depend on the NPS ik $_{i k}$ (as shown in Table 1 and Table 2) and are calculated according to the following formulas:

- $C_{i k}^{1}=-M\left(N \operatorname{Min}_{k}-N P S_{i k}\right) / N M i n_{k}$, gives a bonus to the assignment when worker $i$ has not been performing tasks of type $k$ for the established minimum number of consecutive periods.

- $C_{i k}^{2}=M \min \left[R ; N P S_{i k}+1-N M a x_{k}\right]$, penalises the assignment when worker $i$ has achieved the maximum number of consecutive periods of performing tasks of type $k ; R$ prevents the overflow in the numeric representation of $C_{i k}^{2}$.

- $C_{i k}^{3}=M\left(N P e r_{k}-N P P_{i k}\right) / N P e r_{k}$, penalises the fact that worker $i$ has not achieved the minimum number of consecutive periods without performing tasks of type $k$.

- $C_{i k}^{4}=M\left(N M i n_{k}-N P F_{i}\right) / N M i n_{k}$, penalises the assignment of a task of type $k$ to worker $i$, if the worker has a period of programmed absence that prevents him from reaching the established minimum number of consecutive periods of performing tasks of type $k$.

$$
\begin{aligned}
\cdot C_{i k}^{5} & =\left[\alpha_{i k} \frac{\left\{\left(H_{i}+\beta\right) P T_{i k}-\left(H i s t_{i k}+\beta\right)\right\}^{2}}{\left(H_{i}+\beta\right) P T_{i k}}+\sum_{\forall j \neq k} \alpha_{i j} \frac{\left\{\left(H_{i}+\beta\right) P T_{i j}-H i s t_{i j}\right\}^{2}}{\left(H_{i}+\beta\right) P T_{i j}}\right] \\
& -\sum_{\forall k} \alpha_{i k} \frac{\left\{H_{i} P T_{i k}-H i s t_{i k}\right\}^{2}}{H_{i} P T_{i k}}
\end{aligned}
$$

Where $\frac{\left\{H_{i} P T_{i k}-H i s t_{i k}\right\}^{2}}{H_{i} P T_{i k}}=0$ if $H_{i}=0$. This gives a bonus to or penalises the contribution of the assignment either to approximating or deviating from the ideal $\mathrm{PT}_{i k}$ values. 
Table 1.

Elements of the assignment matrix when $\mathrm{NPS}_{i k}>0$

\begin{tabular}{|l|l|l|}
\hline $\mathrm{NPS}_{i k}>0$ & $\mathrm{NMin}_{k}-\mathrm{NPS}_{i k}>0$ & $\mathrm{NMin}_{k}-\mathrm{NPS}_{i k} \leq 0$ \\
\hline $\mathrm{NPS}_{i k}-\mathrm{NMax}_{k} \geq 0$ & Infeasible & $C_{i k}^{2}$ \\
\hline $\mathrm{NPS}_{i k}-\mathrm{NMax}_{k}<0$ & $C_{i k}^{1}$ & $C_{i k}^{5}$ with $\beta=1$ \\
\hline
\end{tabular}

Table 2.

Elements of the assignment matrix when $\mathrm{NPS}_{i k}=0$

\begin{tabular}{|l|l|l|}
\hline $\mathrm{NPS}_{i k}=0$ & $\mathrm{NPer}_{k}-\mathrm{NPP}_{i k}>0$ & $\mathrm{NPer}_{k}-\mathrm{NPP}_{i k} \leq 0$ \\
\hline $\mathrm{NMin}_{k}-\mathrm{NPF}_{i}>0$ & $C_{i k}^{3}+C_{i k}^{4}$ & $C_{i k}^{4}$ \\
\hline $\mathrm{NMin}_{k}-\mathrm{NPF}_{i} \leq 0$ & $C_{i k}^{3}$ & $C_{i k}^{5}$ with $\beta=\mathrm{NMin}_{k}$ \\
\hline
\end{tabular}

To evaluate the quality of the solutions, we used the function $\widetilde{Z}=\sum_{t=1}^{T} \sum_{i=1}^{W} \sum_{k=1}^{K}\left(P T_{i k}-P R_{i k t}\right)^{2}$, where $\mathrm{PR}_{i k t}\left(\forall i, \forall k \mid f_{C a t_{i}, k}=1\right)$ is the scheduled proportion of worker $i$ 's working time dedicated to performing tasks of type $k$ up to and including period $t$.

As concerns the value of parameter $\beta$, during our first attempt we used $\beta=1$ regardless of the value of NPS $i k$, but a brief computational experiment showed that solutions of a better quality are achieved using $\beta=1$ if $\operatorname{NPS}_{i k}>0$ and $\beta=\mathrm{NMin}_{k}$ if $\mathrm{NPS}_{i k}=0$.

To solve the assignment problems we used the method designed by Jonker and Volgenant (Jonker and Volgenant, 1987), which is considered one of the most efficient out of the available ones (Dell'Amico and Toth, 2000).

\section{Example}

$W=40($ all workers present in every period in time horizon $T) ; C=1 ; K=4 ; F=\left[\begin{array}{llll}1 & 1 & 1 & 1\end{array}\right]$; $D=\left[\begin{array}{llll}20 & 12 & 6 & 2\end{array}\right] \quad($ for all $t)$; 
$P T=\left[\begin{array}{cccc}0.5 & 0.3 & 0.15 & 0.05 \\ \cdots & \ldots & \ldots & \ldots \\ \cdots & \ldots & \ldots & \ldots \\ 0.5 & 0.3 & 0.15 & 0.05\end{array}\right]$

$\alpha_{i k}=1, \forall i k ; T=1000$; considering the following conditions:

NMin $=\left[\begin{array}{llll}2 & 2 & 2 & 2\end{array}\right]$, NMax $=\left[\begin{array}{llll}4 & 4 & 4 & 4\end{array}\right]$ and NPer $=\left[\begin{array}{llll}2 & 2 & 2 & 2\end{array}\right]$, and disregarding possible past activities:

$H=\left[\begin{array}{llll}0 & \ldots & \ldots & 0\end{array}\right]$

and

Hist $=\left[\begin{array}{cccc}0 & 0 & 0 & 0 \\ \cdots & \cdots & \cdots & \cdots \\ \cdots & \cdots & \cdots & \cdots \\ 0 & 0 & 0 & 0\end{array}\right]$.

Fig. 1 represents the percentage of time that one worker dedicates to the four types of tasks over the time horizon. Fig. 2 represents the dedicated percentage of time to tasks of type 2 for a set of six workers over the time horizon.

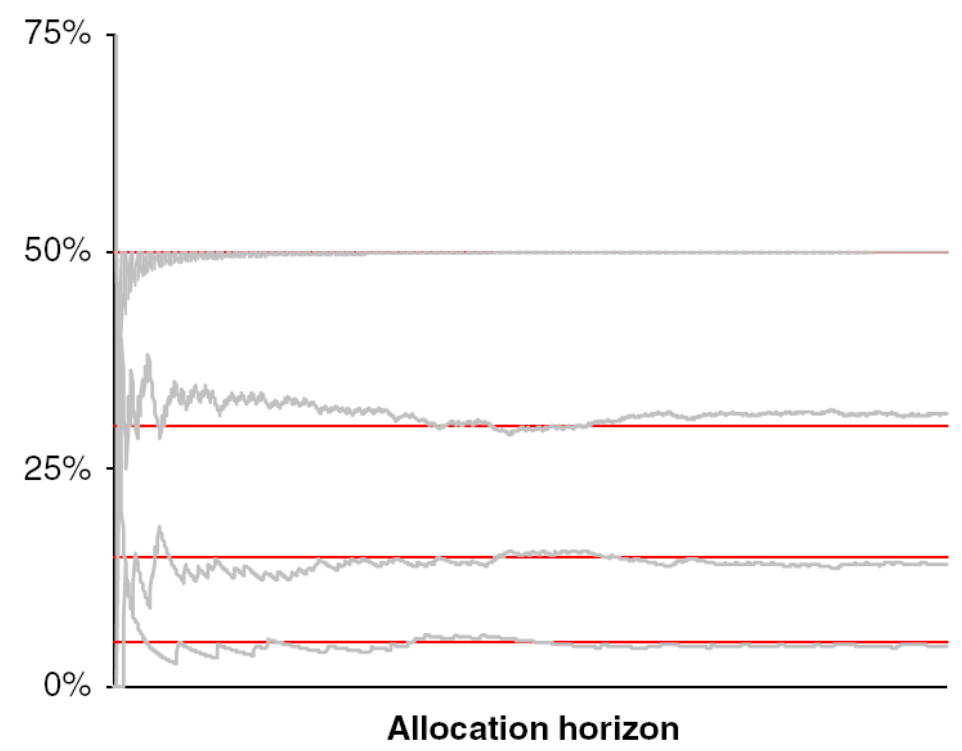

Fig. 1. Percentage of time that one worker dedicates to the four types of tasks. 


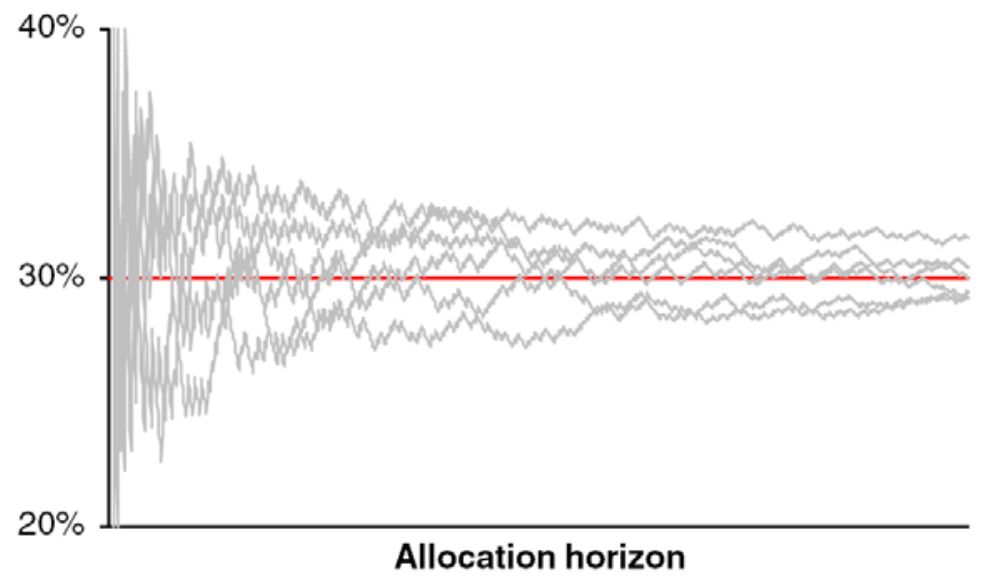

Fig. 2. Percentage of dedicated time by six workers to tasks of type 2 .

For this example, in which $T=1000$, the computing time was equal to $1.68 \mathrm{~s}$, in a PC Pentium 4 at $2.0 \mathrm{GHz}$ with $256 \mathrm{Mb}$ RAM. The solution obtained fulfils the constraints and, as the figures show, the percentages of dedication of the workers to the different types of tasks approaches satisfactorily, after a transient phase, the ideal values.

\section{Computational experiment}

An application in Compaq Visual Fortran 6, that included the Jonker and Volgenant routine, was implemented to generate and solve the sequences of the assignment problems for the instances used in the computational experiment. All the computations were performed using the aforementioned PC.

The values of the parameters used to define the instances for the computational experiment were the following:

$T=$

180 (which may correspond, for instance, to one week of six working days,

from 7:00 to 22:00, and periods of $0.5 \mathrm{~h}$ ), 360,540 or 720 periods.

$|W|=\quad 10,50$ and 100 workers (all present in all the periods of the time horizon).

$(C, K)=(2,3),(3,3)$ and $(4$ categories, 5 types of tasks $)$. 


$$
\begin{aligned}
F_{c k}=\quad & {\left[\begin{array}{lll}
1 & 1 & 0 \\
0 & 1 & 1
\end{array}\right],\left[\begin{array}{lll}
1 & 1 & 1 \\
0 & 1 & 1 \\
1 & 0 & 1
\end{array}\right], } \\
& {\left[\begin{array}{lllll}
1 & 1 & 1 & 1 & 1 \\
0 & 1 & 1 & 1 & 0 \\
1 & 0 & 1 & 0 & 1 \\
0 & 1 & 1 & 1 & 1
\end{array}\right] }
\end{aligned}
$$

according to the values of $C$ and $K$.

$$
\begin{array}{r} 
\\
{\left[\begin{array}{ccc}
0.5 & 0.5 & 0 \\
0 & 0.1 & 0.9
\end{array}\right],\left[\begin{array}{ccc}
0.5 & 0.3 & 0.2 \\
0 & 0.6 & 0.4 \\
0.3 & 0 & 0.7
\end{array}\right],} \\
\mathrm{PT}_{c k}=\left[\begin{array}{ccccc}
0.2 & 0.2 & 0.2 & 0.2 & 0.2 \\
0 & 0.3 & 0.4 & 0.3 & 0 \\
0.3 & 0 & 0.3 & 0 & 0.4 \\
0 & 0.25 & 0.25 & 0.25 & 0.25
\end{array}\right]
\end{array}
$$

according to the values of $C$ and $K$ (the values of PT are assumed to be the same for all the workers in the same category).

$\alpha_{i k}=1, \forall i, k ; H_{i}=0, \forall i ;$ Hist $_{i k}=0, \forall i, k ; \operatorname{NMin}_{k}=4, \forall k ; \operatorname{NMax}_{k}=8, \forall k ; \operatorname{NPer}_{k}=5, \forall k$.

A brief preliminary computational experiment showed that the computing time, as was expected, is proportional to $T$ and that the solution is obtained in a very short time. Subsequently, $T=720$ was adopted for the rest of the experiment.

For each one of the 9 possible combinations of $|W|$ and $(C, K), 10$ instances were obtained by varying the required labour for the diverse types of tasks, $D_{k}$ (although equal for all $t$ ). The information about computing times is summarised in Table 3. 
Table 3.

Minimum $\left(t_{\min }\right)$, average $(\bar{t})$ and maximum $\left(t_{\max }\right)$ computing times (in seconds) corresponding to the nine combinations of $|W|$ and $(C, K)$

\begin{tabular}{|l|l|l|l|l|}
\hline$|W|$ & $(C, K)$ & $\boldsymbol{t}_{\min }$ & $\bar{t}$ & $\boldsymbol{t}_{\max }$ \\
\hline 10 & $(2,3)$ & 0.50 & 0.51 & 0.52 \\
\hline & $(3,3)$ & 0.53 & 0.55 & 0.56 \\
\hline 50 & $(2,3)$ & 1.41 & 1.60 & 1.80 \\
\hline & $(4,5)$ & 0.60 & 0.63 & 0.71 \\
\hline 100 & $(2,3)$ & 3.33 & 4.27 & 5.71 \\
\hline & $(4,5)$ & 3.77 & 2.83 & 3.07 \\
\hline & $(4,5)$ & 11.50 & 12.43 & 13.42 \\
\hline & 3.38 & 3.92 & 4.83 \\
\hline & 3.70 & 3.96 \\
\hline & $(3,3)$ & & \\
\hline
\end{tabular}

As can be seen in Table 3, solving the problem is very fast, even considering the longest computing time (13.42 s), which corresponds to the allocation of one hundred workers in four categories to five types of tasks, with a planning horizon of 720 periods. Moreover, the range of computing times, given $|W|$ and $(C, K)$, is very small. 


\section{Conclusions}

This paper deals with the assignment of tasks to the members of the multi-functional staff of a work center, during each period into which the planning horizon can be divided. For each type of task to perform, all workers who can perform the task do so at equal worker efficiencies. There are constraints that, if possible, should be respected. The objective is that the percentage of working time dedicated by each worker to each type of task be as close as possible to reference values. The problem is modelled as a sequence of assignments, in which appropriate values for the cost matrix depend on the results of the previous assignments.

The obtained results can be considered satisfactory: the solutions meet the constraints; the scheduled percentages steadily approach ideal values, and eventually are very near them; and the calculation times are very short. Therefore, the proposed approach and the corresponding procedure constitute a potential tool for assigning tasks to multi-functional workers in the service industry.

In this work it is assumed that for each type of task to perform, the workers who can perform the task do so at equal worker efficiencies; but, actually, although workers from different categories may be able to perform a specific type of task, habitually certain categories frequently require more time than others do. The future research work will involve relaxing this assumption and, then, solving a more general problem. 


\section{References}

Abernathy et al., 1973 W.J. Abernathy, N. Baloff, J.C. Hershey and S. Wandel, A three-stage manpower planning and scheduling model - a service-sector example, Operations Research 21 (1973), pp. 693-711.

Bellanti et al., 2004 F. Bellanti, G. Carello, F. Della Croce and R. Tadei, A greedy-based neighborhood search approach to a nurse rostering problem, European Journal of Operational Research 153 (2004), pp. 28-40.

Bergman, 1994 R. Bergman, The key is cross-training, Hospitals and Health Network 68 (1994), p. 34.

Buffa et al., 1976 E.S. Buffa, M.J. Cosgrove and B.J. Luce, An integrated work shift scheduling system, Decision Science 7 (1976), pp. 620-630.

Campbell and Diaby, 2002 G.M. Campbell and M. Diaby, Development and evaluation of an assignment heuristic for allocation cross-trained workers, European Journal of Operational Research 138 (2002), pp. 9-20.

Cappanera and Gallo, 2004 P. Cappanera and G. Gallo, A Multicommodity Flow Approach to the Crew Rostering Problem, Operations Research 52 (2004), pp. 583-596.

Corominas and Crespán, 1993 A. Corominas and J. Crespán, Organització del temps de treball, Edicions UPC, Barcelona (1993).

Corominas et al., 2002 A. Corominas, A. Lusa and R. Pastor, Using MILP to plan annualised working hours, Journal of the Operational Research Society 53 (2002), pp. 1101-1108.

Corominas et al., 2004 A. Corominas, A. Lusa and R. Pastor, Planning annualised hours with a finite set of weekly working hours and joint holidays, Annals of Operations Research 128 (2004), pp. 217-233.

Corominas et al., 2005 A. Corominas, J. Ojeda and R. Pastor, Multi-objective allocation of multifunction workers with lower bounded capacity, Journal of the Operational Research Society 56 (2005), pp. 738-743.

Cox, 1989 T. Cox, Towards the measurement of manufacturing flexibility, Production \& Inventory Management Journal First Quarter (1989), pp. 68-72.

Dell'Amico and Toth, 2000 M.P. Dell'Amico and P. Toth, Algorithms and codes for dense assignment problems: the state of the art, Discrete Applied Mathematics 100 (2000), pp. 17 48.

Demerouti et al., 2004 E. Demerouti, S. Geurts, A. Bakker and M. Euwema, The impact of shiftwork on work-home conflict, job attitudes and health, Ergonomics 47 (2004), pp. $987-$ 1002 .

Jonker and Volgenant, 1987 R. Jonker and A. Volgenant, A shortest augmenting path algorithm for dense and sparse linear assignment problems, Computing 38 (1987), pp. 325-340.

Lagodimos and Leopoulos, 2000 A.G. Lagodimos and V. Leopoulos, Greedy heuristic algorithms for manpower shift planning, International Journal of Productions Economics 68 (2000), pp. 95-106. 
Oke, 2000 Oke, A., 2000. Linking human resource flexibility with manufacturing flexibility: enablers of labour capacity flexibility in manufacturing plants. In: Dominguez, J.A., (Ed.), Proceedings of the First World Conference on Production and Operations Management (POM). Sevilla.

Siferd and Benton, 1992 S.P. Siferd and W.C. Benton, Workforce staffing and scheduling: Hospital nursing specific models, European Journal of Operational Research 60 (1992), pp. 233-246.

Tharmmaphornphilas and Norman, 2004 W. Tharmmaphornphilas and B.A. Norman, A quantitative method for determining proper job rotation intervals, Annals of Operations Research 128 (2004), pp. 251-256.

Zülch et al., 2004 G. Zülch, S. Rottinger and T. Vollstedt, A simulation approach for planning and re-assigning of personnel in manufacturing, International Journal of Production Economics 90 (2004), pp. 265-277. 